\title{
Optimizing the Electron Energy for Cryomicroscopy
}

Mathew Peet ${ }^{1}$, Richard Henderson ${ }^{1}$ and Christopher J. Russo ${ }^{1}$

1. MRC Laboratory of Molecular Biology, Cambridge, United Kingdom

Biologically relevant specimens can be imaged in the transmission electron microscope, but electrons also cause damage which limits the information that can be gained. Both the ultimate resolution and the effort required to achieve a particular resolution are therefore determined by the amount of information gained per particle image. This physical limit caused by damage becomes more important as improvements are made in software and hardware for cryomicroscopy.

In recent work [1] we have measured how the elastic and inelastic cross-sections for carbon depend on the accelerating voltage of the electrons, in the energy range typically used in transmission electron microscopy, and made quantitative comparisons to two-dimensional crystals of paraffin and bacteriorhodopsin at $100 \mathrm{keV}$ and $300 \mathrm{keV}$.

By determining the carbon cross-sections for elastic an inelastic scattering (Fig. 1) and measuring the difference in the rates of damage accumulation to the two-dimensional crystals at $300 \mathrm{keV}$ and $100 \mathrm{keV}$, we estimate the potential to gain $25 \%$ more information per unit damage operating at $100 \mathrm{keV}$ rather than $300 \mathrm{keV}$ for typical biological specimen.

Using these measurements, we have introduced an information coefficient which expresses the information gain per unit damage as a function of a specimen thickness and electron energy (Fig. 2). This allows us to determine the optimal energy (accelerating voltage) to use for a given thickness specimen. From these calculations we conclude that $100 \mathrm{keV}$ represents the highest potential for information in single particle cryomicroscopy.

Other limits to reducing the operating energy have already been addressed [2-4], so it seems in the future $100 \mathrm{keV}$ will become the best choice for single particle cryomicroscopy [5].

\section{References:}

[1] M. J. Peet and R. Henderson and C. Russo, Ultramicroscopy, in press https://doi.org/10.1016/j.ultramic.2019.02.007 (2019).

[2] C. J. Russo, R. Henderson, Ultramicroscopy, 187 (2018), p. 43.

[3] C. J. Russo, R. Henderson, Ultramicroscopy, 187 (2018), p. 56.

[4] C. J. Russon, R. Henderson, Ultramicroscopy, 187 (2018), p. 26.

[5] The authors thank Shaoxia Chen, Joanna Brown, Giuseppe Cannone and the LMB EM facility and Jake Grimmett and Toby Darling of LMB Scientific computing for technical support. This work was supported by a Leverhulme Early Career Fellowship to CJR and the Medical Research Council grants MC_UP_1201/17 and MC_U105184322. 
a

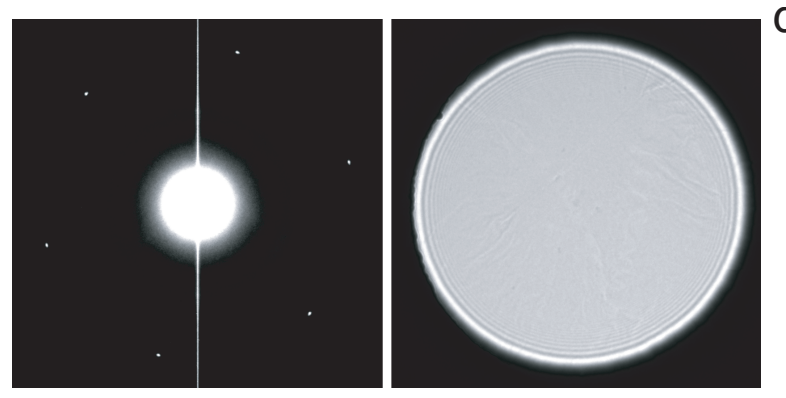

b

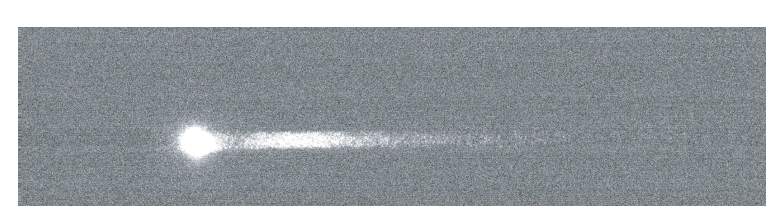

C

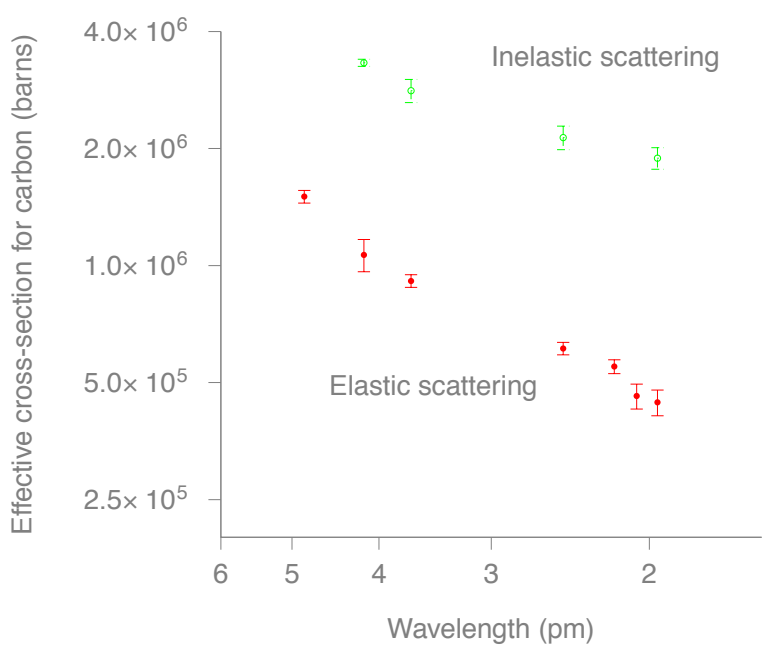

Figure 1. (a) Measurement of elastic, and (b) inelastic cross-sections of the electrons were made as a function of accelerating voltage in the electron microscope (c).

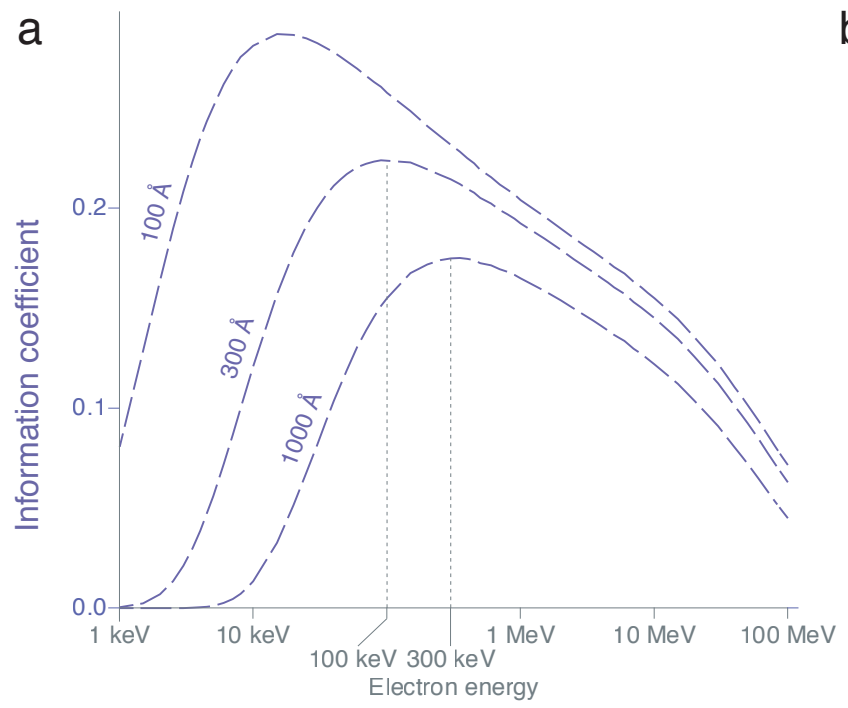

b

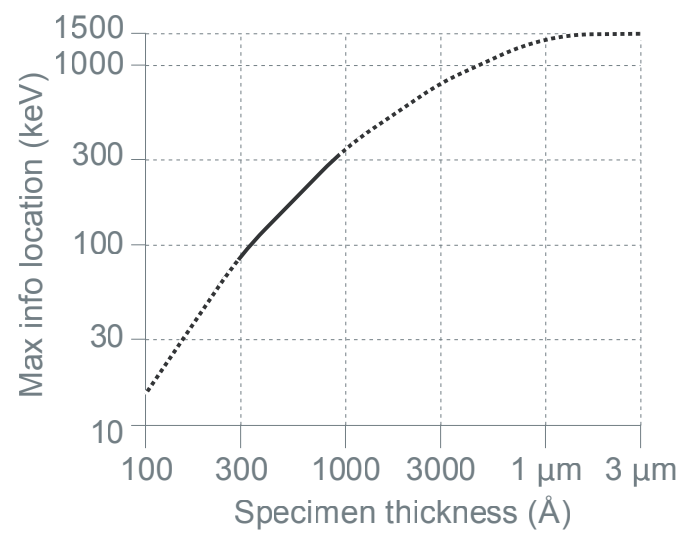

Figure 2. (a) We calculate an Information coefficient as a function of energy and (b) the shift in optimum voltage with specimen thickness. 